\title{
Harmonization of the Land Cover Classification System (LCCS) with the General Habitat Categories (GHC) classification system
}

\author{
Vasiliki Kosmidou ${ }^{a}, *$, Zisis Petrou ${ }^{a}$, Robert G.H. Bunce ${ }^{b}$, Caspar A. Mücher ${ }^{c}$, \\ Robert H.G. Jongman ${ }^{c}$, Marion M.B. Bogers ${ }^{c}$, Richard M. Lucas ${ }^{\mathrm{d}}$, Valeria Tomaselli ${ }^{\mathrm{e}}$, \\ Palma Blonda ${ }^{\mathrm{f}}$, Emilio Padoa-Schioppa ${ }^{\mathrm{g}}$, Ioannis Manakos ${ }^{\mathrm{a}}$, Maria Petrou ${ }^{\mathrm{a}}$ \\ a Information Technologies Institute (ITI), Centre for Research and Technology Hellas, 6th km Harilaou - Thermi, P.0. Box: 60361, 57001 Thessaloniki, Greece \\ ${ }^{\mathrm{b}}$ Estonian University of Life Sciences, Kreutzwaldi 1, Tartu 51014, Estonia \\ ${ }^{\mathrm{c}}$ Alterra Wageningen UR, Droevendaalsesteeg 3, 6700 AA Wageningen, The Netherlands \\ d Institute of Geography and Earth Sciences, Aberystwyth University, Aberystwyth, Ceredigion, SY23 2EJ Wales, UK

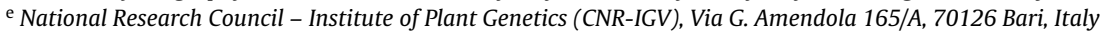 \\ ${ }^{\mathrm{f}}$ National Research Council - Institute of Intelligent Systems for Automation (CNR-ISSIA), Via Giovanni Amendola, 122/D-I, 70126 Bari, Italy \\ ${ }^{g}$ University of Milano-Bicocca, Department of Sciences of Environment and Territory, RULE - Research Unit of Landscape Ecology, Piazza della Scienza 1, \\ 20126 Milano, Italy
}

\section{A R T I C L E I N F O}

\section{Article history:}

Received 13 March 2013

Received in revised form 25 July 2013

Accepted 26 July 2013

\section{Keywords:}

Habitat

Land cover

Biodiversity monitoring

Remote sensing

Plant life forms

Strategic survey

\begin{abstract}
A B S T R A C T
Monitoring land cover and habitat change is a key issue for conservation managers because of its potential negative impact on biodiversity. The Land Cover Classification System (LCCS) and the General Habitat Categories (GHC) System have been proposed by the remote sensing and ecological research community, respectively, for the classification of land covers and habitats across various scales. Linking the two systems can be a major step forward towards biodiversity monitoring using remote sensing. The translation between the two systems has proved to be challenging, largely because of differences in definitions and related difficulties in creating one-to-one relationships between the two systems. This paper proposes a system of rules for linking the two systems and additionally identifies requirements for site-specific contextual and environmental information to enable the translation. As an illustration, the LCCS classification of the Le Cesine protected area in Italy is used to show rules for translating the LCCS classes to GHCs. This study demonstrates the benefits of a translation system for biodiversity monitoring using remote sensing data but also shows that a successful translation is often depending on the degree of ecological knowledge of the habitats and its relationship with land cover and contextual information.
\end{abstract}

(C) 2013 Elsevier Ltd. All rights reserved.

\section{Introduction}

A key challenge in conserving biodiversity, designing strategic biodiversity conservation plans and reporting on their success, is the establishment of a reliable and constant monitoring of changes in land cover and understanding the implications on habitat quality and extent and the flora and fauna contained. At national, regional and global levels, organizations have responded to the increasing urgency for regular updates on land cover and habitat information (Schmeller, 2008). However, this has led to an increasing number of datasets that have used different classification schemes and mapping methods. This has resulted in incompatibility of these datasets for a range of applications (Herold et al., 2006). For this reason,

\footnotetext{
* Corresponding author. Tel.: +30 2311 257758; fax: +30 2310474128 .

E-mail address: kosmidou@iti.gr (V. Kosmidou).
}

the Group of Earth Observations (GEO) is pursuing global harmonization in all "Societal Benefit Areas" - including biodiversity to facilitate its monitoring. This process has been stimulated by the reporting obligations for the Convention on Biological Diversity (CBD), which requires assessments of the status and trends of species, habitats and ecosystems (GEO BON, 2011).

Recent developments of Earth Observation (EO) systems and capacity to build integrated databases have significantly increased the ability to coordinate and exchange knowledge on environmental state and change of stocks at a global level. Although the potential of EO data to biodiversity monitoring has been recognized (Nagendra, 2001; Turner et al., 2003; Groom et al., 2006; Mücher, 2011), there is still no systematic protocol for its use at global, continental and regional scales (Buchanan et al., 2008). Whilst many schemes (e.g., the COoRdination of INformation on the Environment - CORINE and the Land Cover Classification System - LCCS) have been developed for remotely mapping of Land Cover and Land 
Use ( $\mathrm{LC} / \mathrm{LU}$ ) across a range of scales, the different methods used (even within countries) lead to difficulties in creating land cover change maps (Hazeu et al., 2011; Scholes et al., 2012) and hence its translation into habitat categories. For this reason, observation and classification systems need to be harmonized to support environmental management at national, regional and local scale. Where more than one system is used, the relationships between the entities of these systems need to be made explicit (Scholes et al., 2012). Additionally, the translation of remotely sensed land cover maps into habitat maps is extremely important, as habitats better link to flora and fauna species and are therefore considered significant as indicators of biodiversity (Bunce et al., 2012, 2013a,b).

The need for such a translation system is becoming urgent because of recognized declines in global biodiversity, which is mainly due to habitat destruction and degradation through land cover changes (Hansen et al., 2004; Sala et al., 2000). Such changes have been occurring at an increasing rate over recent centuries and decades and have placed increasing pressures on landscapes, habitats and biodiversity in general. Hence, the monitoring of changes in $\mathrm{LC} / \mathrm{LU}$ is central to monitoring biodiversity change. Land cover differs from land use in that it is an expression of a specific land use intervention (including no intervention at all) on a specific type of land at a specific point in time (Stomph et al., 1997). More specifically, the term 'land use' refers both to the way the land is used (i.e., manipulated by the intention of humans) and to the use or economic function that the land has to humans (i.e., the purpose of the intervention). The term 'land cover' defines 'the attributes occupying a part of the Earth's surface, such as vegetation, artificial constructions, rocks and water, which can be distinguished from a distance' (Anderson et al., 1976). Hence, in principle, everything that is observed remotely (e.g., by a satellite sensor) can be regarded as the land cover. In many cases the land use can be inferred from the land cover through virtue of its spatial configuration and context. As an example, sports fields can be distinguished from grassland by their specific geometry and their location within urban areas, while the urban areas themselves can be observed remotely. However, many-to-many relationships exist between land cover and land use and hence they should always be used as separate terms.

The high demand for standardized and consistent LC/LU datasets motivated the Food and Agriculture Organization of the United Nations (FAO) and the United Nations Environment Programme (UNEP) to introduce the LCCS (Di Gregorio and Jansen, 1998, 2005). This system is intended to be capable of capturing any land cover throughout the world, independent of specific applications and/or geographical areas. The LCCS is also intended to overcome problems associated with the interpretation of different land cover class definitions. This is because it defines a set of independent diagnostic criteria strictly based on vegetation physiognomy and structure rather than establishing other land cover classes based on terminology. These criteria are then used to define land cover classes, compatible with any definition and allowing for relations with existing classifications and labels (Di Gregorio and Jansen, 2005; Jansen, 2010). The system is both hierarchical and clearly constructed and has been proven a valid tool in describing land cover and detecting change (Bartholomé and Belward, 2005).

Land cover maps are often derived from remote sensing data along with ancillary geographic information (e.g., soil maps), and other data derived from remote sensing data (e.g., Digital Elevation Models (DEMs), Environmental Zones) can be integrated to obtain information on the spatial distributions of habitats (Jongman et al., 2011; Mücher et al., 2009; Mücher, 2011). Where generated, these datasets provide a basis for quantifying and analysing changes in habitats and provide information to respond to the reporting obligations according to Article 17 of the EU Habitats Directive (92/43
EEC). However, as with land cover classification, the framework for habitat monitoring needs to be consistent in contrasting landscapes and also applicable worldwide. Habitat is a widely used term but, as with land cover, the content of the concept 'habitat' remains diverse, ambiguous and difficult to use consistently for monitoring purposes (Bunce et al., 2013a). Common approaches for monitoring changes in habitat also require definitions and rules that are harmonised continentally and globally. To address this problem, Bunce et al. (2005, 2006, 2008, 2011) introduced the General Habitat Categories (GHCs) and tested it throughout Europe. The GHC procedure has been developed based on the definition of habitat as "an element of land that can be consistently defined spatially in the field in order to determine the principal environments in which organisms live". The GHC method therefore comes as an ecological refinement of the land cover categorization used in LCCS (Bunce et al., 2011). The basis of the GHCs is the classification of plant life forms (LFs) introduced by the Danish ecologist Raunkiaer early in the 20th century. LFs are determined by the position of buds during unfavourable season (e.g., cold or dry seasons). The composition of LFs and the relative abundance in the biological spectra are a direct link among vegetation, flora anatomy and environmental (mainly climate) variables. Raunkiaer (1934) demonstrated that LFs are correlated with the main environmental gradient from the equator to the arctic. Different studies showed that the model that Raunkiaer applied to global level can be applied consistently to regional or local context (Küchler and Zonneveld, 1988; Bunce et al., 2011). Hence, the recording of vegetation structure is central to the GHC methodology and also facilitates interaction between the GHC and LCCS taxonomies. Furthermore, LCCS categories extracted from satellite-derived land cover maps can then be translated into GHC habitat maps as both use LFs, height and cover as distinctive criteria, thereby provide a direct link between remote sensing and in situ observations. The GHC methodology includes the use of "qualifiers" (LF qualifiers, Environmental qualifiers, Site qualifiers, Management qualifiers). In particular, Environmental, Site and Management qualifiers provide useful and detailed information on some environmental factors such as soil moisture and reaction, site morphology and on type and level of human activity. This information is essential to achieve a detailed level of habitat type and to detect possible habitat changes. Also the LCCS procedure provides a wide set of environmental and technical attributes, by means of which it is possible to achieve the most detailed level of land cover description, the closest to the level of habitat. The objective of this work, conducted under the auspices of the EU-funded BIO_SOS project (www.biosos.eu), is to describe, for the first time, a framework for a robust and consistent translation of LCCS classes to GHC classes. Based on this framework, the BIO_SOS project aims to develop a pre-operational system for cost effective and timely monitoring of changes in land cover and habitats within and along the borders of protected areas, and judge the effectiveness in protecting and conserving the regions from human impacts. Input data sources are satellite-based measurements and ancillary data collected in situ; the basic output products of the BIO_SOS project include maps of land cover and habitat based on the LCCS and GHC taxonomies and biodiversity indicators. In this framework, precise awareness of the potential and limitations of each classification scheme and the correlations between the two taxonomies within the BIO_SOS, EO Data for Habitat Mapping (EODHAM) system is essential. Recently, Tomaselli et al. proved the strong interconnection between the LCCS and GHC taxonomies in comparison to other LC/LU and habitat classification systems (Tomaselli et al., 2013). In this paper the aim is to further elaborate the relationships between the two taxonomies in order to identify specific mapping rules. The proposed framework has been applied to the Le Cesine Natura 2000 site in Italy. 


\section{Methods}

\subsection{Study area}

The study area used in this paper is the Le Cesine site, a coastal wetland situated in the south-east of Italy in the region of Puglia with an area of 348 ha (see Fig. 1 in the supplementary material). Le Cesine is one of the oldest protected areas in Puglia, declared as a Ramsar site in 1977 and a State Natural Reserve since 1980, and falls within the Natura 2000 network as proposed Site of Community Importance (pSCI, IT9150032) and Special Protection Area (SPA, IT9150014). In particular, the study area comprises natural terrestrial and aquatic vegetation of trees, shrubs and herbaceous, cultivated fields and urban areas (see Fig. 2 in the supplementary material). The humid area is one of the most important in southern Italy, formed by two large coastal lagoons and various channels, marshes and wet grasslands. Within the Reserve, the Cladium mariscus (L.) Pohl communities (priority habitat $7210^{*}$ according to the Habitats Directive) are frequently subject to fires, sometimes arising from the adjacent agricultural areas and sometimes from arsons. In addition, marine erosion has caused a progressive reduction of the sandbank separating the coastal lagoons from the sea and, in some cases, the break of the sandbank. There has also been a progressive increase of the salinity of the lagoon water, with a corresponding spread of halophytic communities. Finally, agricultural practices taking place both inside and outside the protected area are likely to lead to the disappearance of temporary ponds, which are priority habitats according to the Habitats Directive. In general, this Natura 2000 site is not only exposed to a conversion to agricultural practices, but also to illegal urban development and to an increasing tourist pressure (Herold et al., 2009). A LCCS map for the site was produced by translating a pre-existing CORINE land cover map obtained by photo interpretation of an aerial image dated 2005 and validated by in-field campaigns carried out in 2007-2008 (Tomaselli et al., 2013).

\subsection{Principles of the LCCS and the GHC taxonomies}

The LCCS is the result of an initiative towards an internationally agreed reference base for land cover (Di Gregorio and Jansen, 2005, 1998). One of the basic principles adopted in the LCCS approach is that a given land cover class is defined by the combination of a set of independent diagnostic attributes, the so-called classifiers (e.g., life form, height, cover, etc.). This approach allows the use of the most appropriate indicators and reduces the total number of classes by removing impractical combinations of classifiers. An increased detail in the description of a land cover feature is linked to the increase in the number of classifiers used. In other words, the more classifiers added, the more detailed the subsequent class is (Di Gregorio and Jansen, 2005). The class boundary is then defined by the different number of classifiers. Thus, emphasis is no longer on the class name, but on the set of classifiers used to define this class.

In the LCCS, the land cover classes are identified in two phases, with a dichotomous key used initially to define eight major land cover types based on three classifiers, namely the presence of vegetation, edaphic condition and artificiality of cover.

These eight land cover super categories are:

- A11.Cultivated and Managed Terrestrial Areas.

- A12.Natural and Semi-Natural Terrestrial Vegetation.

- A23.Cultivated Aquatic or Regularly Flooded Areas.

- A24.Natural and Semi-Natural Aquatic or Regularly Flooded Vegetation.

- B15.Artificial Surfaces and Associated Areas.

- B16.Bare Areas.
- B27.Artificial Water Bodies, Snow and Ice.

- B28.Natural Water Bodies, Snow and Ice.

The definitions of these LCCS super categories are given in detail in the FAO-LCCS handbook (Di Gregorio and Jansen, 2005) and have been applied to several parts of the world (Jansen and Di Gregorio, 2003).

The initial phase is followed by the Modular-Hierarchical phase, in which land cover classes are generated by a combination of sets of pre-defined classifiers (i.e., pure land cover classifiers (e.g., life form, spatial aspect, etc.), environmental attributes and specific technical attributes), facilitating a more detailed description of land cover classes and reducing the likelihood of impractical combinations of classifiers (Di Gregorio and Jansen, 2005). The basic rules governing the concepts of the LCCS classification concludes with the definition of LF and dominance.

The GHC classification system (Bunce et al., 2011) resulted from several EU projects, such as the BIOHAB-a framework for the coordination of biodiversity and habitats (www.edinburgh.ceh.ac.uk/biota/biohab_page.htm) and the European Biodiversity Observation Network (EBONE, http://www.ebone.wur.nl), concerning surveillance and monitoring of biodiversity through habitats. The objective of the GHC classification is to provide the lowest common denominator for European habitats and serve as a primary structure for recording ecosystems or habitats, in an easy but consistent manner. The GHC system is designed specifically for linking in situ and remote sensing observations, and detecting and mapping changes in habitats. The system applies stringent criteria to ensure that real change is recorded and results are not distorted by differences in definitions, between observers or in recording techniques (Bunce et al., 2008, 2011). The procedure has been designed for strategic survey of habitats and is based on a set of stratified random samples.

Similarly to LCCS, the GHC classification is based on a restricted number of super-categories, with these being urban (URB), cultivated (CUL), sparsely vegetated (SPV), trees and shrubs (TRS), herbaceous wetland (HER) and other herbaceous (HER) (Bunce et al., 2011). GHCs are characterized by LFs, leaf type, phenology, height and cover and are detailed by qualifiers on the environment, site, management and species composition (Bunce et al., 2008; Raunkiaer, 1934). Sparsely vegetated (bare) land has been refined by adding Non-Life-Form habitats (NLF) to complete the system, as rock, stones, sand and gravel are considered habitats in their own right, especially in deserts and mountains, where they do represent different species assemblages (Olsvig-Whittaker et al., 2011).

As the GHC classification is used in situ, the field map is first separated into elements (i.e., areal, linear and point elements) according to their size and dimension, as defined by the Minimum Mappable Element (MME) rules with a threshold of $400 \mathrm{~m}^{2}$ and Minimum Mappable Length (MML) rules with a threshold of $5 \mathrm{~m}$ (Bunce et al., 2011). The GHC codes and all possible combinations within each of the super categories can be given to any mapping element. No other combinations are possible than those reported in the Handbook (Bunce et al., 2011). For Europe, a maximum of 160 GHCs is described and the global list is expected to consist of about 700 GHCs.

In both systems, LFs are defined by their physiognomic aspect and provide direct links between in situ vegetation and nonvegetation structure, and dynamic global vegetation models (e.g., Sitch et al., 2003) and, therefore, they can be used to classify both in situ data and the patterns present on satellite images. A condition of height is applied to separate trees from shrubs, i.e. woody plants higher than $5 \mathrm{~m}$ are classified as trees, whereas those below $5 \mathrm{~m}$ are classified as shrubs. In LCCS, this general rule is subjected to the exception that a woody plant with a clear physiognomic aspect 
of trees can be classified as a tree even if its height is lower than $5 \mathrm{~m}$, but more than $3 \mathrm{~m}$.

Concerning the concept of dominance which determines the class assigned, both classifications use the cover of the upper canopy layer as the main criterion. This means that the dominant layer is the tree canopy followed by the shrub and the herbaceous one. Additional vegetation layers can be added in the stratification. Some limitations in the use of this classifier have been introduced to avoid class combinations that are irrelevant from the structural point of view (Di Gregorio and Jansen, 2005). In LCCS, the cover categories are used to determine the dominant LF, whether Closed (over 65\%), Open (15-65\%) or Sparse LF (4-15\% cover). The cover categories in the GHC classification have been designed to fit in with previous work, especially in the Mediterranean literature, but have a comparable division with two percentage rules: over $70 \%$ for single habitats or $40-60 \%$ for mixed habitats. If there are equal proportions of LFs, then precedence rules are provided. Other qualifiers can also be added to express variations between elements that may have the same GHC and identify the details of the habitat type, e.g., additional LF qualifiers, environmental qualifiers, global codes, site qualifiers and management qualifiers. The complete list of the qualifiers is provided in the Manual (Bunce et al., 2011) and is used to further refine the habitat recording.

\subsection{Linking the LCCS and GHC taxonomies}

In order to create a framework for the translation of LCCS classes into GHC classes in the most accurate way, the correspondence between the two taxonomies needs to be established. Difficulties in the translation between the two taxonomies exist because of differences in definitions and interpretations of the landscape, even though both have been designed to meet the fundamental attributes for global vegetation classification (Running et al., 1995). In particular, both LCCS and GHC employ land cover and land use, although emphasizing different aspects. However, as both classifications have been developed independently by different scientific communities, the definitions of some LFs in LCCS differ from that adopted by the GHC framework even at the land cover level. The main differences between the two classifications can be found in the terminology, the criteria of height in vegetation categories, the hierarchy that leads to the first level of categories and the rules relating to the percentage of vegetation cover. Moreover, the detail of information and the scale of remote sensing observations might induce additional one-to-many or many-to-one relationships as outlined next.

\subsubsection{Terminology discrepancies}

The LCCS is based on published classification legends of various definitions with most common the Küchler and Zonneveld (1988). The GHC classification is based on LFs, as first described by Raunkiaer (1934), and as later adapted by various authors such as Küchler (1967), Mueller-Dombois and et Ellenberg (1974), Box (1981), and even later by Küchler and Zonneveld (1988). This can be illustrated by using the example of herbaceous. LCCS divides herbaceous into two main categories, i.e., forbs and graminoids. A further height criterion is considered mandatory in the LCCS, whereas in the GHC taxonomy the height information for herbaceous is reported as an additional optional qualifier. Instead, in GHC, the herbaceous LFs are divided into categories relating mainly to location of the bud, seasonality and plant types, i.e., helophytes $(H E L)$, leafy hemicryptophytes ( $L H E)$, etc. The reason for this is the importance of these characteristics for the identification of biodiversity and the restriction of the first version of the GHC taxonomy to Europe. However, this results in intrinsic discrepancies between the two taxonomies that need to be resolved to avoid one-to-many and many-to-many mapping rules. For instance, the therophytes class (THE) of the GHC taxonomy corresponds to annual plants that survive during unfavourable season as seeds (Bunce et al., 2011), which in the LCCS terminology is translated as either annual forbs or graminoids (one-to-two correspondence). More specifically, species such as Aira praecox L., which is in the THE category, is an annual grass belonging to the family of Poaceae and, therefore, it would be recorded as annual graminoid in LCCS. On the other hand, the Viola arvensis Murray, which is also designated with the THE code in GHC, is an annual plant (e.g., Violaceae) and would be recorded as an annual forbs in LCCS. However, as these species are unlikely to cover an area over $400 \mathrm{~m}^{2}$, they will not appear as a mapped unit. This can however happen to reeds (e.g., Phragmites australis (Cav.)Trin. ex Stend., Arundo donax) that can reach over $2 \mathrm{~m}$ height. They can be perennial and they are treated as the life form qualifier of bamboos and canes in the GHC classification.

Another distinguishing example of the discrepancies in definitions is the use of the term 'needle-leaved' in the LCCS, referring to trees and shrubs of the botanical group Gymnospermae (FordRobertson, 1971), carrying typical needle-shaped leaves. This category includes all plants with needle-like leaves which are strictly conifers, whereas gymnosperms contain a wide range of leaf type structures. GHC uses the term coniferous including only a subclass of the plants described as needle-leaved in the LCCS taxonomy. So, the one-to-one relationship between the corresponding LCCS and GHC classes in this case would not be feasible. The adaptation made to the LCCS to use the term conifer makes this one-to-one crosswalk feasible again. In addition, the dominant species and their coverage, recorded in column 5 of the field study sheets in the GHC procedure (Bunce et al., 2011), enable the correct allocation to be made later to the LCCS class where the terms used often need interpretation according to strict botanical definitions. In the GHC Manual (Bunce et al., 2011) botanical terms are carefully used. For example, some genera such as Podocarpus, have organs which appear to be leaves but are morphologically modified shoots. In order to cover this, the Manual emphasises that if the organs look like leaves, they should be treated as such.

\subsubsection{Height discrepancies}

The criteria of height of vegetation in the definition of LCCS and GHC classes are listed in Table 1. In particular, the height ranges defined by the GHC for chamaephytes and phanerophytes do not correspond exactly with those defined by LCCS for trees and shrubs. Moreover, LCCS defines different ranges of height for herbaceous types, whereas these ranges are not provided in GHC. These discrepancies most likely arise from the differences in end-user requirements. For example, GHC has been set-up from an ecological perspective, has rules related to strict botanical definitions and has originally a Europe-oriented approach for biodiversity surveillance and monitoring, while LCCS was developed as a system for land cover and land use mapping using remote sensing with focus on Africa. These discrepancies and different perspectives on the landscape must be taken into consideration, when designing the framework for the transition from LCCS to GHC maps.

\subsubsection{Principle discrepancies}

As a result of the different perspectives, basic concepts of land use are considered in a different manner within the two taxonomies, resulting in some discrepancies, most of which can be resolved through database management. A typical example is the top-category "A11.Cultivated and Managed Terrestrial Areas" of the LCCS classification. This class refers to areas where the natural vegetation has been removed and replaced by other types of vegetation that require human activities to be maintained. At first glance, this class would be considered in a one-to-one relation with the Cultivated (CUL) class of the GHC taxonomy. For instance, an olive grove, which corresponds to "broadleaved 
Table 1

Vegetation height definitions in the LCCS and GHC taxonomies.

\begin{tabular}{|c|c|c|}
\hline Height (m) & LCCS & GHC \\
\hline$>40$ & A12.A3.B5 Trees & Giga phanerophytes, GPH \\
\hline $14-40$ & & Forest phanerophytes, FPH \\
\hline $7-14$ & A12.A3.B6 Trees & \\
\hline $5-7$ & A12.A3.B7 Trees & \\
\hline $3-5$ & A12.A3.B7 Trees/A12.A4.B8 Shrub & Tall phanerophytes, TPH \\
\hline $2-3$ & A12.A4.B9 Shrub & Mid phanerophytes, MPH \\
\hline \multicolumn{3}{|l|}{$0.6-2$} \\
\hline $0.3-0.6$ & A12.A4.B10 Shrub & Low phanerophytes, LPH \\
\hline $0.05-0.3$ & & Shrubby chamaephytes, SCH \\
\hline$<0.05$ & & Dwarf chamaephytes, DCH \\
\hline
\end{tabular}

evergreen trees, orchards" in the LCCS (A11 A1.A7.A9.W8) will be apparently mapped as cultivated woody crops (CUL/WOC) in the GHC. In a special case though, that olive grove fields are used for parking, the land use would change the assigned class in the GHC classification and would instead be recorded within the urban trees (URB/TRE) class. Additionally, in recreation areas, the natural vegetation might be replaced by other vegetation, i.e., needle-leaved plantations of Pinus sylvestris L. in Estonia and Pinus pinaster Aiton in France, which are tapped to extract turpentine. In the LCCS they would be recorded as "needle-leaved evergreen tree crops plantation" (A11 A1.A8.A9.W7), whereas in the GHC taxonomy these would more likely be considered a forest category and would be recorded in the TRS class. Hence, the mapping between the LCCS and GHC taxonomies in the ostensibly simple class of the cultivated and managed areas is not always a one-to-one relation. Qualifiers recorded could be used to obtain exact correspondence. However, the application of the species data recorded in the GHC procedure can make subsequent allocation more precise.

\subsubsection{Precedence rule discrepancies}

In terms of vegetation cover, the LCCS taxonomy requires that vegetation cover exceeds $4 \%$ of the area in order to be assigned to the primary vegetated area class, whilst the GHC requires over $30 \%$ cover, except for deserts, where the actual percentage is used. These definitions could lead to differences in relating the results between these two schemes. Both taxonomies make use of the upper canopy layer and the percentage of vegetation cover, but at a more detailed level many discrepancies do exist. A representative example of this would be a natural area consisting of $80 \%$ herbaceous-forbs and $20 \%$ cover of dispersed trees. According to the upper canopy layer rule in the LCCS, this area would be recorded as A12.A3 A11 "Open Trees (15-65\%)" with the stratification/layering qualifier of forbs. On the contrary, according to the percentage rule in the GHC, the percentage of trees is not adequate enough to be recorded as the primary vegetation, so precedence would be given to the lower vegetation. In particular, it would be recorded within the HER category and the trees could be included as a point element with the appropriate qualifier (i.e., open-OPE) (Bunce et al., 2011). Additional information recorded for each GHC as described by Bunce et al. (2011) could be elaborated with database management to obtain one-to-one agreement.

\subsubsection{Difficulties due to detail of information}

When only pure land cover classifiers are used to define the LCCS classes, several GHC classes can be contained within one LCCS class and hence it is difficult to relate the two classifications even at the life form level. Therefore, in order to facilitate the translation, the LCCS map must contain adequate detail of information. For instance, if the vertical stratification qualifier of the LCCS was not recorded in the previous example, the transition to the correct GHC would be impossible. Thus, the relation between the two classifications is susceptible to the degree of information available in both systems. Moreover, some classes (e.g., water) can also be considered to be both artificial and natural. For example, straight channels (e.g., for freshwater or brackish water) are artificial but often link to the natural system (e.g., estuaries or meandering streams). Artificial pools may be constructed for recreation, for water storage or to restore and eventually become part of a 'natural' system (e.g. an active raised bog in Wales). The concepts of land cover and land use are often confusing; hence some compromises need to be made when linking the two taxonomies.

\subsubsection{Scale discrepancies}

The GHC has been developed to facilitate consistent habitat monitoring within field studies (scale of approximately 1:5000), whereas the LCCS has been developed with a remote sensing perspective from regional to global scales (i.e., it is scale independent, Di Gregorio and Jansen, 1998, 2005). Therefore the relationship between the two classification schemes is dependent upon the scale and hence the spatial resolution of satellite or airborne systems. In particular, linear elements, which are especially important to biodiversity in managed landscapes (Bunce et al., 2008), might not be considered as individual elements in the LCCS classification, but as part of adjacent classes. In many cases, the spatial resolution of the satellite sensor needs to be high to very high (2-20 m resolution) in order to allow the discrimination of LCCS classes. Information on vegetation height (e.g., as derived from airborne LiDAR data) may become essential. In some environments (e.g., the active raised bog of Cors Fochno in mid Wales), a complex mosaic of shrubs, graminoids and lichens occurs and several LCCS classes might be assigned to this habitat depending on the spatial resolution of the observing sensor. The LCCS map has to contain as homogeneous classes as possible in order to make the translation to GHC accurate, robust and meaningful. Therefore, in complex landscapes with many land cover variations, an LCCS map with a larger number of small area classes is preferred to a map with a smaller number of large area classes. The former will result in more discriminative GHC classes through more accurate relationships.

\subsection{Framework for translating LCCS classes into GHC classes}

Although the two taxonomies have been developed sharing common concepts and converge highly compared with other classifications (Tomaselli et al., 2013), the discrepancies described above need to be smoothed out in order to achieve harmonization between them. As previously explained, a major contradiction occurs in the percentage of vegetation cover. The EO-based measurement of the percentage of vegetation cover for determining the LCCS classes is per se a very difficult task, with high error variance, posing fuzziness in the selection of the correct class, especially for the least occurring classes (Vanden Borre et al., 2011). This can be perceived as the reliability of the measurement. Under this notion, it is advised to ignore this discrepancy between the two taxonomies, when the percentage of vegetation cover is around $20 \%$ 
and use the scattered (SCA) and OPE global qualifier codes of the GHC taxonomy (Bunce et al., 2011) in the case of percentages of tree cover lower than $10 \%$. In the latter, if the stratification qualifier in the LCCS is obtained, it can be used to identify the GHC code. Alternatively, the output of the dominant land cover according to LCCS should be considered to identify the habitat.
Following the tree-like structure of the LCCS classification scheme, the relations between the two taxonomies were built based on their comparison on a theoretical level by ecologist experts, as depicted in Figs. 1-4. The mapping is extended to the LF level considering LCCS qualifiers i.e., height, leaf type, leaf phenology, etc. Examining the mapping relations, one can observe that
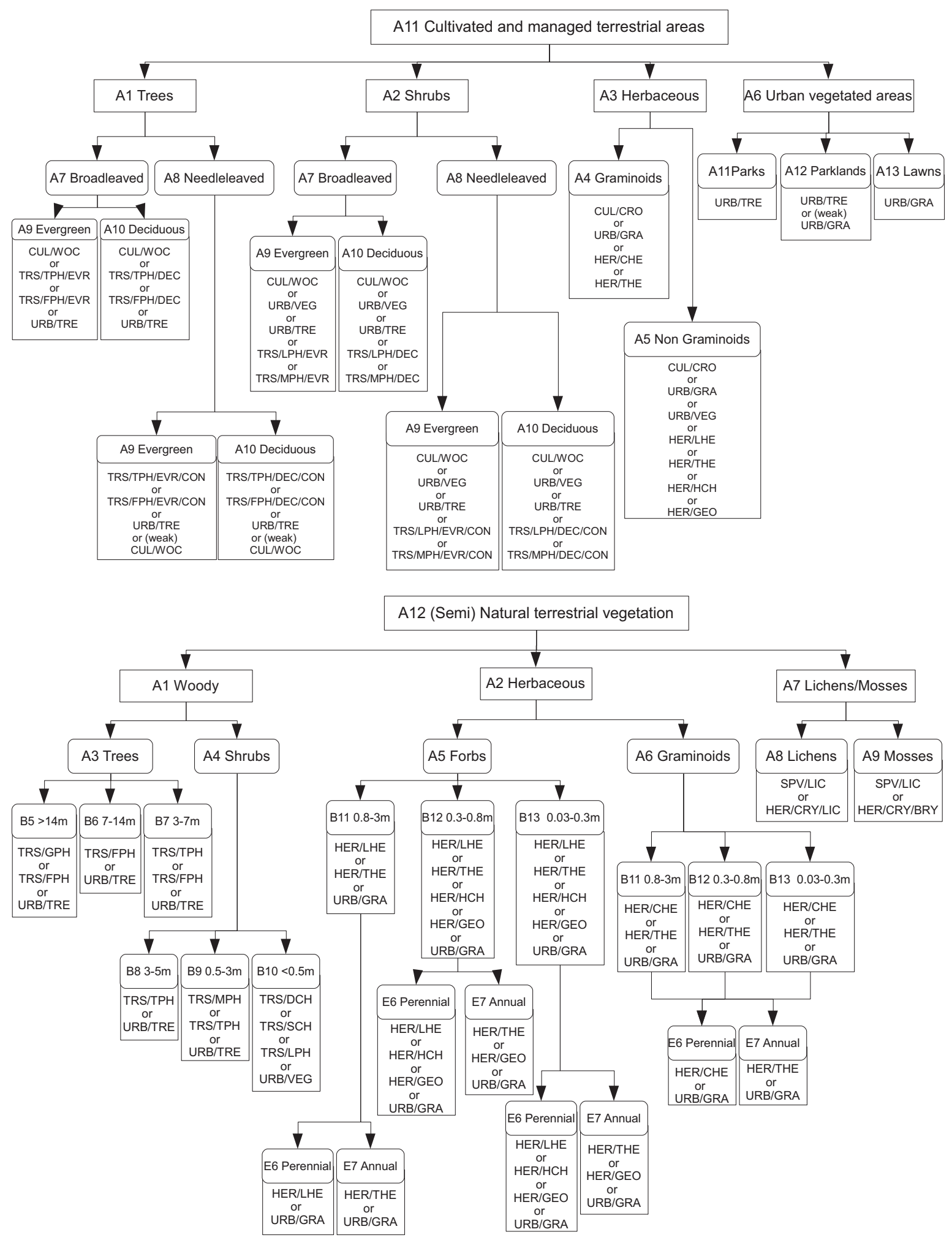

Fig. 1. The mapping rules between the A11 and A12 LCCS categories and the corresponding GHC classes. 
there are several one-to-many matching in the vegetated categories, mainly due to the discrepancies in the definitions between the two taxonomies, as described earlier. A representative example is the case of a garden with fruit trees, such as black currents and gooseberries, less than $3 \mathrm{~m}$ tall. This would be denoted with the label "A11.A2 cultivated shrubland" in the LCCS taxonomy, while taking the land use into consideration through context, it would be assigned to $C U L / W O C$, if it is in agricultural land use or URB/TRE, when classified as an urban garden. So, one single LCCS class could correspond to two GHC classes. In most cases, an orchard will be classified as $C U L / W O C$, as the area of an urban garden most likely would not meet the MME criterion. It is evident that this one-tomany mapping could be resolved by considering spatial relations, e.g., urban garden is likely to be near a building.

In addition to the land use, the height discrepancies increase the ambiguity. For instance, considering the strict definitions of height categories, the LCCS "A12.A4.A10.B3.B9 medium/high shrubland" (B9 refers to height between 0.5 and $3 \mathrm{~m}$ as shown in Table 1 ) could be either tall phanerophytes (TPH) (2-5 m) or mid phanerophytes $(M P H)(0.6-2 \mathrm{~m})$ or, in an extreme case, even low phanerophytes $(L P H)(0.3-0.6 \mathrm{~m})$, with MPH being the most probable one. The $L P H$ class can be excluded though, taking into account the reliability of the measurement of height. This reveals the urgent need to take into consideration the probability of appearance and the reliability of measurements of phenological and structural details, when translating LCCS maps into GHC maps. Therefore, some mapping relations are denoted as "weak" (see Fig. 1), revealing a low probability of such a translation to occur.

It is important to note that both LCCS and GHC classifications share common LF and NLF general categories. However, it is inevitable that even at the NLF case, inconsistencies occur that can lead to one-to-many relations. For example, gravel is defined as "coarse fragments having a size less than $6 \mathrm{~cm}$ " within the LCCS taxonomy, whereas in the GHC as having a diameter less than $5 \mathrm{~cm}$. Such details cannot be resolved using remote sensing data and only a coarse assignment of the land cover class is possible. Strictly based on the definitions and not taking into consideration the capability of a remote sensing system, the "B16.A1 A14 gravel" class (LCCS) will correspond to the gravel (GRV) or the stones (STO) class (GHC), assigning a very low probability to the latter. Taking into consideration the uncertainty in the measurement of the size and the ecological importance of such a distinction, the "B16.A1 A14 gravel" would correspond to GRV alone (Fig. 3).

The relations that provide the framework for translating LCCS classes into GHC classes, presented in Figs. 1-4, can be considered as expert mapping rules integrated into a rule-based system.
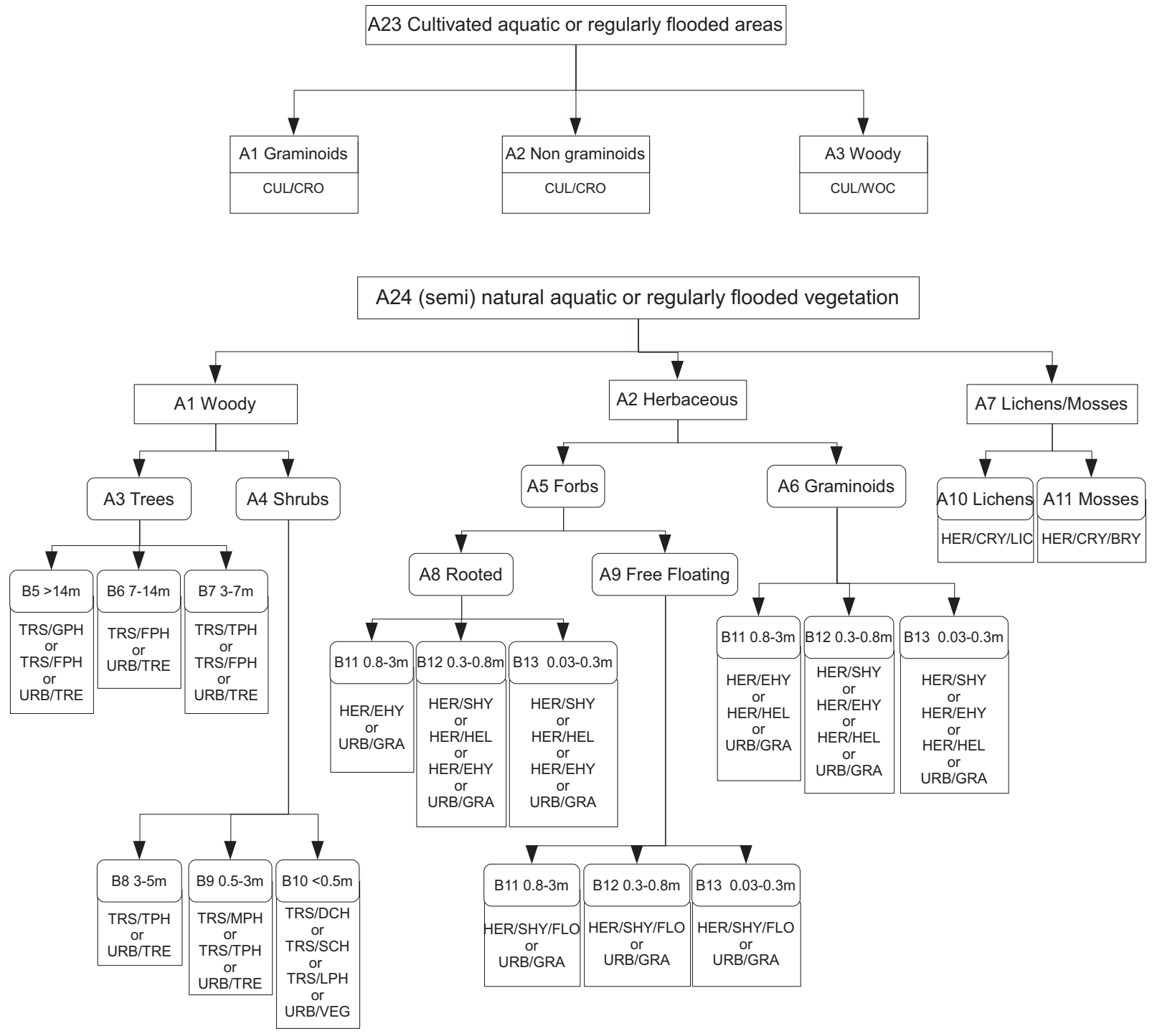

Fig. 2. The mapping rules between the A23 and A24 LCCS categories and the corresponding GHC classes. 

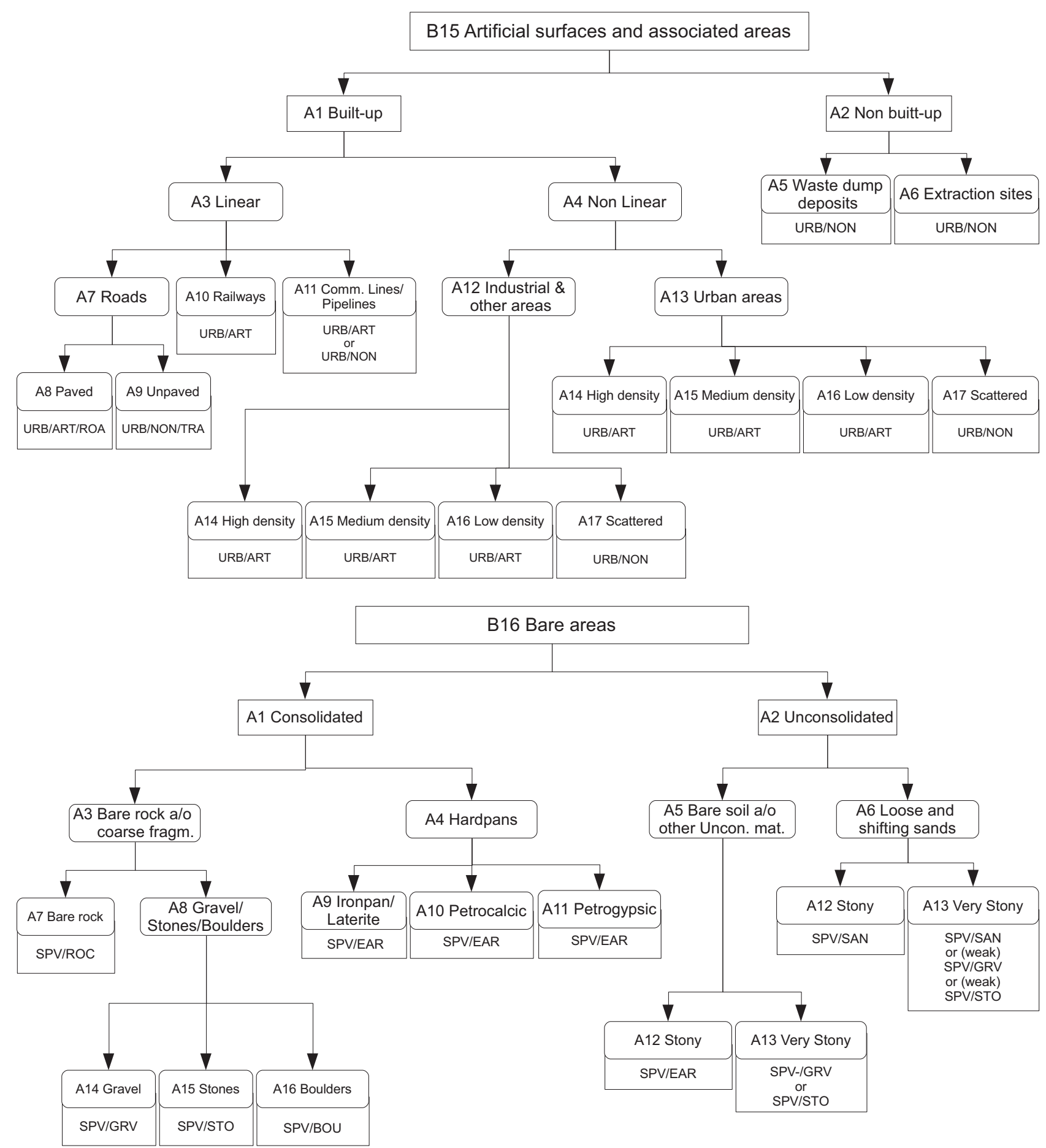

Fig. 3. The mapping rules between the B15 and B16 LCCS categories and the corresponding GHC classes.

This system has been built on decision trees following the tree-like structure of rules within the BIO_SOS project. Each LCCS class is represented by the root of a decision tree, whose leaves are all the possible GHC habitat classes that correspond to the specific land cover class.

\section{Results and discussion}

The framework has been tested in the study area of the Le Cesine site, in which a variety of land cover classes is present. Using the above described mapping rules, the corresponding GHC map was produced (see Fig. 3, in supplementary material). Table 2 provides the full list of the LCCS classes present in Le Cesine site and their translation to the corresponding GHC classes. The results show uncertainty revealing the need of additional data on land use for individual patches in the area. For example, the "A24.A5.A13.B4.C2.E5_B13.E7 Open annual short forbs vegetation on temporarily flooded land", could be either emergent hydrophytes $(E H Y)$, submerged hydrophytes (SHY), HEL or urban herbaceous (GRA) in the GHC system, depending on the land use and the LFs present following the general expert rules (see Fig. 2). Taking into consideration that in the Le Cesine site the vegetated 

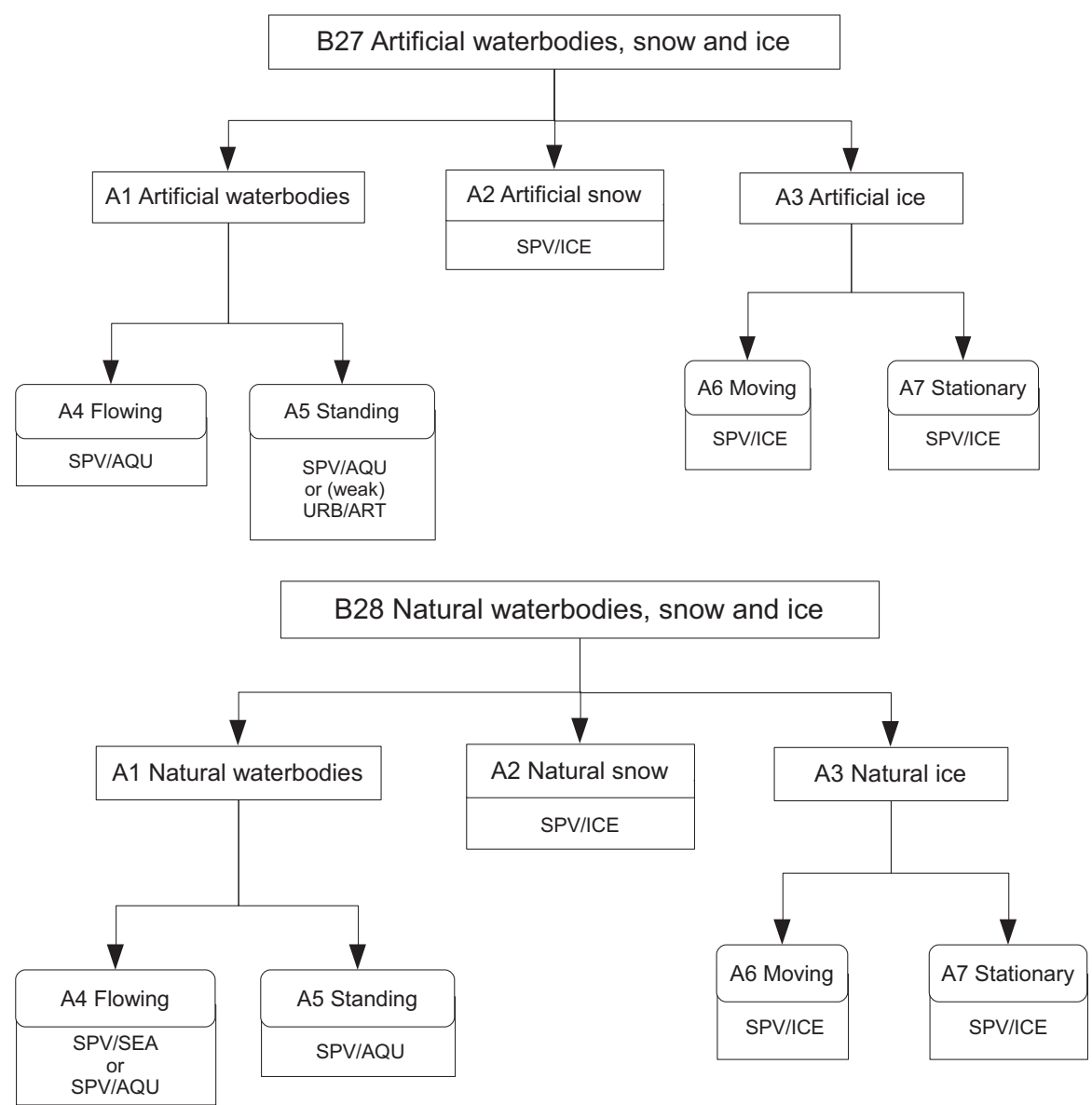

Fig. 4. The mapping rules between the B27 and B28 LCCS categories and the corresponding GHC classes.

ponds are not within urban area, the mapping to the class GRA will be excluded. Additionally, the level of detail of the LCCS classes plays a crucial role in the improvement of the translation into the GHC classes. The A12.A6 grassland in LCCS corresponds to both caespitose hemicryptophytes ( $C H E$ ) and THE GHC classes, but when providing ecological information details through the additional classifiers " $E 6$ " for perennial, then the matching is limited to the CHE class only. Finally, canopy height models, e.g., derived from

Table 2

The LCCS classes and the translation to GHC classes present in the test site of Le Cesine.

\begin{tabular}{|c|c|c|}
\hline LCCS class & LCCS class description & GHC class \\
\hline A11.A1.B1.C1.D1-W7_A8.A9.B3 & Rainfed needleaved evergreen tree crops-plantations & TRS/FPH/EVR/CON or TRS/TPH/EVR/CON \\
\hline A11.A1.B2.C1.D1-W7_A8.A9 & $\begin{array}{l}\text { Small sized field of rainfed needleaved evergreen tree } \\
\text { crops-plantations }\end{array}$ & TRS/FPH/EVR/CON or TRS/TPH/EVR/CON \\
\hline A11.A1.B1.C1.D1-W7_A8.A9.B4 & Rainfed needleaved evergreen tree crops-plantations & TRS/FPH/EVR/CON or TRS/TPH/EVR/CON \\
\hline A11.A1.B1.C1.D1-W8_A7.A9.B3 & Rainfed broadleaved evergreen tree crops-orchard & CUL/WOC or URB/TRE \\
\hline A11.A1.B2.C1.D1-W8_A7.A9 & $\begin{array}{l}\text { Small sized field of rainfed broadleaved evergreen tree } \\
\text { crops-orchard }\end{array}$ & CUL/WOC or URB/TRE \\
\hline A11.A5.B2.C2.D3 & $\begin{array}{l}\text { Less than } 2 \text { ha size fields of irrigated no-graminoid crops- one } \\
\text { additional crop }\end{array}$ & $\begin{array}{l}\text { CUL/CRO or HER/GEO or HER/HCH or HER/LHE or } \\
\text { HER/THE or URB/GRA or URB/VEG }\end{array}$ \\
\hline A12.A4.A10.B3.D1.E1_B9 & Broadleaved evergreen open medium-high shrublands & TRS/MPH/EVR or TRS/TPH/EVR or URB/TRE \\
\hline A12.A4.A11.B3.D1.E1_B10 & Broadleaved evergreen open dwarf shrublands & $\begin{array}{l}\text { TRS/DCH/EVR or TRS/SCH/EVR or TRS/LPH/EVR or } \\
\text { URB/VEG }\end{array}$ \\
\hline A12.A4.A10.B3.D1.E2_B9 & Broadleaved deciduous medium-high closed shrubland & TRS/MPH/DEC or TRS/TPH/DEC or URB/TRE \\
\hline A12.A4.A10.B3.D2.E1_B9 & Needleaved evergreen medium-high closed shrubland & TRS/MPH/CON or TRS/TPH/CON or URB/TRE \\
\hline A12.A5.A11.B4.E5_A13.B13.E7 & Open annual short forbs & HER/THE or HER/GEO or URB/GRA \\
\hline A12.A5.A11.B4.E5_B13.E7 & Open annual short forbs & HER/THE or HER/GEO or URB/GRA \\
\hline A12.A5.A10.B4.E5_B12.E7 & Closed annual medium-tall forbs & HER/THE or HER/GEO or URB/GRA \\
\hline A12.A6.A11.B4.E5_B12.E6 & Open perennial medium-tall grasslands & HER/CHE or URB/GRA \\
\hline A24.A4.A12.B3.C2.D3_B10 & Aphyllous closed dwarf shrubs on temporarily flooded land & TRS/DCH or TRS/SCH or TRS/LPH or URB/VEG \\
\hline A24.A5.A13.B4.C2.E5_B13.E7 & Open annual short forbs on temporarily flooded land & HER/SHY or HER/EHY or HER/HEL or URB/GRA \\
\hline A24.A5.A16.B4.C1.E5_A15.B12.E6 & Perennial sparse medium-tall forbs on permanently flooded land & HER/SHY or HER/EHY or HER/HEL or URB/GRA \\
\hline A24.A6.A12.B4.C2.E5_B11.E6 & Perennial closed tall grassland on temporarily flooded land & HER/SHY or HER/EHY or HER/HEL or URB/GRA \\
\hline A24.A6.A12.B4.C2.E5_B12.E6 & $\begin{array}{l}\text { Perennial closed medium-tall grasslands on temporarily flooded } \\
\text { land }\end{array}$ & HER/SHY or HER/EHY or HER/HEL or URB/GRA \\
\hline B15.A1.A4.A13.A17 & Scattered urban or other areas & URB/NON \\
\hline B15.A3.A8 & Paved roads & URB/ART/ROA \\
\hline
\end{tabular}


LiDAR data, can be used to discriminate between the different height categories of shrubs and trees to reduce inconsistency in the correspondence of the two taxonomies. Thus, even though the "A12.A4.A10.B3.D1.E2_B9 broadleaved deciduous medium-high closed shrubland" based on the expert rules (see Fig. 1) would correspond to either TRS/MPH/DEC, TRS/TPH/DEC or URB/TRE, the information of height would help to exclude one of the TRS/MPH/DEC and TRS/TPH/DEC classes. Therefore, it is evident that additional data can resolve the ambiguity in the one-to-many relations linking the two taxonomies.

Examining the results, the URB class appears to cover a greater extend than the actual structure of the site. The main difficulty arises from the need of more explicit information on land use. For instance, some olive groves are used as parking lots in the test site. Therefore, instead of being mapped as WOC, these fields correspond to the TRE class, because in habitat terms they are no longer active olive plantations. However, due to the spatial resolution of the image and its acquisition date, cars parked under olive trees cannot be easily detected in the image. Management qualifiers, included in the GHC procedure, can provide essential information on type and level of human activity, in order to solve such ambiguities. In general, qualifiers provide useful and detailed information on environmental conditions and on the type of management. The inclusion of such additional information will help to resolve the ambiguity in the translation of LCCS classes into the corresponding GHCs and hence into the ecological corresponding habitats in the landscape. Qualifiers are appropriate for the detection of changes occurring in the studied area and can be therefore used as effective indicators both of environmental condition and of management type.

For habitat mapping it is also important to know the drivers of change and the pressures on biodiversity that are recorded in the land use and management qualifiers used within the GHC mapping procedure. Such information could be integrated in refined rules, i.e., rules on land use or adjacency to certain habitats.

\section{Conclusions}

In conclusion, this paper presents a framework for the translation of LCCS classes into GHC classes to facilitate the harmonization of land cover and habitat datasets towards consistent monitoring and evaluation of conservation management. The intrinsic characteristics of the two taxonomies are examined in detail to identify their correspondences. Discrepancies in terminology, height criteria, precedence rules and basic principles induce a number of one-to-many relations, revealing the need of additional information. The inclusion of land use and management information and the use of additional qualifiers, such as species, offers a high level of detail to the class description and can resolve the ambiguities to a certain extent. Information on management type and the major species for each GHC could be utilized to improve relationships.

Applying the proposed framework to other sites will facilitate the refinement of the linking relations between the two taxonomies, employing real-data and therefore moving the translation from the strictly theoretical level to real-life scenarios.

\section{Acknowledgements}

The work presented in this paper was carried out as part of the European Union's Seventh Framework Programme FP7/2007-2013, SPA.2010.1.1-04: "Stimulating the development of GMES services in specific area”, under grant agreement 263435 , project BIO_SOS: BIOdiversity Multi-Source Monitoring System: from Space To Species (http://www.biosos.eu), coordinated by
CNR-ISSIA, Bari-Italy and the 7th Framework Project EBONE under Contract Nr: ENV-CT-2008-212322.

\section{Appendix A. Supplementary data}

Supplementary data associated with this article can be found, in the online version, at http://dx.doi.org/10.1016/ j.ecolind.2013.07.025.

\section{References}

Anderson, J.R., Hardy, E.E., Roach, J.T., Witmer, R.E., 1976. Land use and land cover classification system for use with remote sensor data. U.S. Geological Survey Professional Paper, 964

Bartholomé, E., Belward, A.S., 2005. GLC2000: a new approach to global land cover mapping from earth observation data. Int. J. Remote Sens. 26 (9), 1959-1977.

Box, E.O., 1981. Macroclimate and Plant Forms: An Introduction to Predictive Modeling in Phytogeography. Tasks for Vegetation Science, vol. 1. Dr. W. Junk BV, Den Haag, pp. 258.

Buchanan, G.M., Nelson, A., Mayaux, P., Hartley, A., Donald, P.F., 2008. Delivering a global, terrestrial, biodiversity observation system through remote sensing. Conserv, Biol. 23, 499-502.

Bunce, R.G.B., Bogers, M.M.B., Evans, D., Halada, L., Jongman, R.H.G., Mücher, C.A., Bauch, B., de Blust, G., Parr, T.W., Olsvig-Whittaker, L., 2013a. The significance of habitats as indicators of biodiversity and their link to species. Ecol. Ind., http://dx.doi.org/10.1016/j.ecolind.2012.07.014.

Bunce, R.G.B., Bogers, M.M.B., Evans, D., Jongman, R.H.G., 2013b. Field identification of habitats directive Annex I habitats as a major European biodiversity indicator. Ecol. Ind., http://dx.doi.org/10.1016/j.ecolind.2012.10.004.

Bunce, R.G.H., Bogers, M.M.B., Ortega, M., Morton, D., Allard, A., Prinz, M., Peterseil, J., Elena-Rossello, J., Jongman, R.H.G., 2012. Conversion of European habitat data sources into common standards. Wageningen, Alterra, AlterraReport, 2277.

Bunce, R.G.H., Bogers, M.M.B., Roche, P., Walczak, M., Geijzendorffer, I.R., Jongman, R.H.G., 2011. Manual for Habitat and Vegetation Surveillance and Monitoring: Temperate, Mediterranean and Desert Biomes, 1st ed. Wageningen, Alterra Report 2154.

Bunce, R.G.H., Metzger, M.J., Jongman, R.H.G., Brandt, J., de Blust, G., Elena-Rossello, R., Groom, G.B., Halada, L., Hofer, G., Howard, D.C., Kovàř, P., Mücher, C.A., PadoaSchioppa, E., Paelinx, D., Palo, A., Perez Soba, M., Ramos, I.L., Roche, P., Skånes, H., Wrbka, T., 2008. A standardized procedure for surveillance and monitoring European habitats and provision of spatial data. Landsc. Ecol. 23, 11-25.

Bunce, R.G.H., Pérez Soba, M., Gomez-Sanz, V., Garcia del Barrio, J.M., Elena-Rossello, R., 2006. European framework for surveillance and monitoring of habitats: a methodological approach for Spain. Invest. Agrar.: Sist. Recur. For. 15 (3), 249-261.

Bunce, R.G.H., Groom, G.B., Jongman, R.H.G., Padoa-Schioppa, E., 2005. Handbook for Surveillance and Monitoring of European Habitats, 1st ed. Wageningen, Alterra Report 1219.

Di Gregorio, A., Jansen, L.J.M., 2005. Land Cover Classification System (LCCS): Classification Concepts and User Manual for Software Version 2. FAO Environment and Natural Resources Service Series, No. 8, Rome.

Di Gregorio, A., Jansen, L.J.M., 1998. Land Cover Classification System (LCCS): Classification Concepts and User Manual. For software version 1.0. GCP/RAF/287/ITA Africover - East Africa Project in Cooperation with AGLS and SDRN. Nairobi, Rome.

Ford-Robertson, E.C., 1971. Terminology of Forest Science, Technology Practice and Products. Society of Amer Foresters, Washington DC.

GEO BON 2011. Adequacy of Biodiversity Observation Systems to Support the CBD 2020 Targets. Available from: http://www.earthobservations.org/ documents/cop/bi_geobon/2011_cbd_adequacy_report.pdf (assessed April 2012).

Hansen, A.J., deFries, R.S., Turner, W., 2004. Land use change and biodiversity: a synthesis of rates consequences during the period of satellite imagery. In: Gutman, G., Janetos, A.C., Justice, C.O., Moran, E.F., et al. (Eds.), Land Change Science: Observing, Monitoring and Understanding Trajectories of Change on the Earth's Surface. Series title Remote Sensing and Digital Image Processing, vol. 6. Publisher Kluwer Academic Publishers, pp. 277-300.

Groom, G., Mücher, C.A., Ihse, M., Wrbka, T., 2006. Remote sensing in landscape ecology: experiences and perspectives in a European context. Landsc. Ecol. 21 (3 Spec. Iss.), 391-408.

Hazeu, G.W., Bregt, A.K., de Wit, A.J.W., Clevers, J.G.P.W., 2011. A Dutch multi-date land use database: identification of real and methodological changes. Int. J. Appl. Earth Observ. Geoinform. 13, 682-689.

Herold, M., Hubald, R., Di Gregorio, A., 2009. Translating and Evaluating Land Cover Legends Using the UN Land Cover Classification System (LCCS). Global Observation of Forest and Land Cover Dynamics, GOFC-GOLD Report No. 43. Jena, Germany.

Herold, M., Woodcock, C., Di Gregorio, A., Mayaux, P., Belward, A.S., Latham, J. Schmullius, C.C., 2006. A joint initiative for harmonization and validation of land cover datasets. IEEE Trans. Geosci. Remote Sens. 44 (7), 1719-1727. 
Jansen, L.J.M., 2010. Analysis of Land Change with Parameterised Multi-level Class Sets Exploring the Semantic Dimension. Wageningen University, pp. 245 (PhD thesis).

Jansen, L.J.M., Di Gregorio, A., 2003. Land-use data collection using the "land cover classification system": results from a case study in Kenya. Land Use Policy 20, 131-148.

Jongman, R.H.G., Bouwma, I.M., Griffioen, A., Jones-Walters, L., Van Doorn, A.M.M. 2011. The Pan European Ecological Network - PEEN. Landsc. Ecol. 26 (3), 311-326.

Küchler, A.W., 1967. Vegetation Mapping. Ronald Press, New York,

Küchler, A.W., Zonneveld, I.S., 1988. Handbook of Vegetation Science. Kluwer Academic Publishers, Dordrecht, the Netherlands.

Mücher, C.A., Hennekens, S.M., Bunce, R.G.H., Schaminée, J.H.J., Schaepman, M.E., 2009. Modelling the spatial distribution of Natura 2000 habitats across Europe. Landsc. Urban Plan. 92 (2), 148-159, http://dx.doi.org/10.1016/j.landurbplan.2009.04.003.

Mücher, C.A., 2011. Monitoring biodiversity using remote sensing and field surveys. In: Trisurat, Y. Shrestha, R.P., Alkemade, R. (Eds.), Land Use, Climate Change and Biodiversity Modeling: Perspectives and Applications. , pp. 78-102.

Mueller-Dombois, D., et Ellenberg, H., 1974. Aim and Methods of Vegetation Ecology. Wiley, New York.

Nagendra, H., 2001. Review article. Using remote sensing to assess biodiversity. Int. J. Remote Sens. 22 (12), 2377-2400.

Olsvig-Whittaker, L., Frankenberg, E., Magal, Y., Shkedy, Y., Amir, S., Walczak, M., Luck-Vogel, M., Jobse, D., de Gelder, A., Blank, L., Carmel, Y., Levin, N., HarariKremer, R., Blankman, D., Boeken, B., 2011. EBONE in Mediterranean and Desert Sites in Israel, with Additional Notes on South Africa Report on Field Tests in LTER Sites and Habitat Monitoring Alterra Report 2260.

Raunkiaer, C., 1934. The Life Forms of Plants and Statistical Plant Geography, Being the Collected Papers of C. Raunkiaer. Oxford University Press, Oxford.
Running, S.W., Loveland, T.R., Pierce, L.L., Nemani, R.R., Hunt, E.R., 1995. A remote sensing based vegetation classification logic for global land cover analysis. Remote Sens. Environ. 51 (1), 39-48.

Sala, O.E., Chapin, F.S., Armesto, J.J., Berlow, E., Bloomfield, J., Dirzo, R., HuberSanwald, E., Huenneke, L.F., Jackson, R.B., Kinzig, A., Leemans, R., Lodge, D.M. Mooney, H.A., Oesterheld, M., Poff, N.L.R., Sykes, M.T., Walker, B.H., Walker, M. Wall, D.H., 2000. Global biodiversity scenarios for the year 2100. Science 287 (5459), 1770-1774.

Schmeller, D.S., 2008. European species and habitat monitoring: where are we now? Biodivers. Conserv. 17, 3321-3326.

Scholes, R.J., Walters, M., Turak, E., Saarenmaa, H., Heip, C.H.R., O'Tuama, E., Faith, D.P., Mooney, H.A., Ferrier, S., Jongman, R.H.G., Harrison, I.J., Yahara, T., Pereira, H.M., Larigauderie, A., Geller, G., 2012. Building a global observing system for biodiversity. Curr. Opin. Environ. Sust. 4, 139-146.

Sitch, S., Smith, B., Prentice, I.C., Arneth, A., Bondeau, A., Cramer, W., Kaplan, J.O. Levis, S., Lucht, W., Sykes, M.T., Thonicke, K., Venevsky, S., 2003. Evaluation of ecosystem dynamics, plant geography and terrestrial carbon cycling in the LPJ dynamic global vegetation model. Global Change Biol. 9 (2), 161-185.

Stomph, T.J., Mücher, C.A., Fresco, L.O., 1997. Environmental impact of land use: a new basis for analysis. Land 1.2, 129-142.

Tomaselli, V.M., Dimopoulos, P., Marangi, C., Kallimanis, A., Adamo, M., Tarantino, C. Panitsa, M., Terzi, M., Veronico, G., Lovergine, F., Nagendra, H., Lucas, R., Mairota, P., Mucher, S., Blonda, P., 2013. Translating land cover/land use classifications to habitat taxonomies for landscape monitoring: a mediterranean assessment. Landsc. Ecol. 28 (5), 905-930.

Turner, W., Spector, S., Gardiner, N., Fladeland, M., Sterling, E., Steininger, M., 2003. Remote sensing for biodiversity science and conservation. Trends Ecol. Evol. 18 (6), 306-314

Vanden Borre, J., Paelinckx, D., Mücher, C.A., Kooistra, L., Haest, B., De Blust, G. Schmidt, A., 2011. Integrating remote sensing in Natura 2000 habitat monitoring: prospects on the way forward. J. Nat. Conserv. 19, 116-125. 